\title{
FASCIOLASIS HEPATICA: REPORTE DE UN CASO Y REVISION DE LA LITERATURA
}

\author{
Drs. Alejandra López $P^{(1)}$, Claudio Silva $F^{(2)}$, David Busel $M^{(1)}$. \\ 1. Médico Radiólogo. 2. Médico Becado Radiología \\ Centro de Imagenología. Hospital Clínico, Universidad de Chile.
}

\begin{abstract}
Human fasciolasis is a zoonosis linked to the trematod Fasciola hepatica, which affects primarily bovine and ovine cattle, and by chance, humans. Difficulty in clinical diagnosis due to its various patterns of presentation, it may be necessary to obtain images that enable a diagnosis. Therefore, in endemic zones (such as Latin America, Asia, and northern Africa), should include this entity as differential diagnosis for focal liver lesion, especially in the presence of eosinophilia.
\end{abstract}

Key words: Fasciolasis, Focal liver lesion.

Resumen: La fasciolasis humana es una zoonosis causada por la Fasciola hepática, trematodo que en su vida adulta afecta principalmente el ganado ovino y bovino, siendo el hombre huésped accidental. La dificultad en el diagnóstico clínico, dada su variada sintomatología, muchas veces requerirá del apoyo imagenológico, por lo que el radiólogo debe estar preparado y conocer la epidemiología de este patógeno, especialmente en aquellas zonas endémicas (Latinoamérica, zonas de Asia y el Norte de África). Es importante considerarla dentro del diagnóstico diferencial de lesiones focales hepáticas, especialmente en el contexto clínico de eosinofilia.

Palabras claves: Distomatosis, Fasciolasis, Lesión focal hepática.

\section{Introducción}

La fasciolasis humana es una zoonosis parasitaria causada por la Fasciola hepática, trematodo que en su vida adulta afecta principalmente el ganado bovino y caprino, siendo el hombre un huésped accidental ${ }^{(1)}$. Se estima que

López A. Fasciolasis hepática: Reporte de un caso y revisión de la literatura. Rev Chil Radiol 2004; 10: 118-123.

Correspondencia: Dra. Alejandra López P.

Centro de Imagenología, Hospital Clínico

Universidad de Chile. Santos Dumont 999 - Sector D

Independencia, Región Metropolitana - Chile su presencia es endémica en todo Latinoamérica, y en Chile se extiende de la I a la XI Región, con tasas de infectividad de ganado de $30.1 \%$ para bovinos, $14 \%$ en caprinos, y $12.3 \%$ de equinos ${ }^{(2)}$. Se ha descrito prevalencia de infección humana de alrededor de un $0.7 \%$ en Linares, VII Región ${ }^{(2)}$, lo que ha llevado a algunos autores a inferir que en Santiago debieran ocurrir entre 20 a 30 casos sintomáticos al año ${ }^{(3)}$, muchos de los cuales nunca son diagnosticados correctamente.

Se presenta el caso de una paciente afectada por esta patología y se discute su etiopatogenia, clínica y hallazgos en imágenes, destacando la importancia de su conocimiento por el radiólogo para sospechar su diagnostico.

\section{Caso clínico}

Paciente de 44 años, del sexo femenino, con antecedentes de colecistectomía. Presenta historia de dolor abdominal vago en hemiabdomen superior durante una semana, asociado a vómitos, afebril, sin diarrea ni coluria, que no cede a antiespasmódicos habituales; motivo por el cual se hospitaliza durante 7 días, realizándose exámenes de laboratorio, donde destaca bilirrubina en rango normal, SGOT $26.6 \mathrm{U} / \mathrm{L}$, SGPT $33 \mathrm{U} / \mathrm{L}$, fosfatasas alcalina de 219, Hemograma con 8900 leucocitos y $24 \%$ eosinófilos (recuento absoluto: 2136).

Se efectuó una ecotomografía abdominal (US) que fue interpretada como normal y tomografía computada con contraste endovenoso (TC) que demostró una lesión focal hipodensa heterogénea en segmentos VI-VII, que se interpretó como sugerente de hemangioma o hiperplasia nodular focal (Figura 1). Se da de alta, con tratamiento sintomático, indicándose control con hematólogo dado la eosinofilia.

Con estos antecedentes, es derivada al Servicio de Hematología de nuestro hospital, donde se solicita un nuevo hemograma que revela $45.7 \%$ de eosinófilos (recuento absoluto: 3943). Se repitió US (Figura 2) y se efectuó resonancia magnética de hígado solicitada para estudio de "Tumor hepático" 

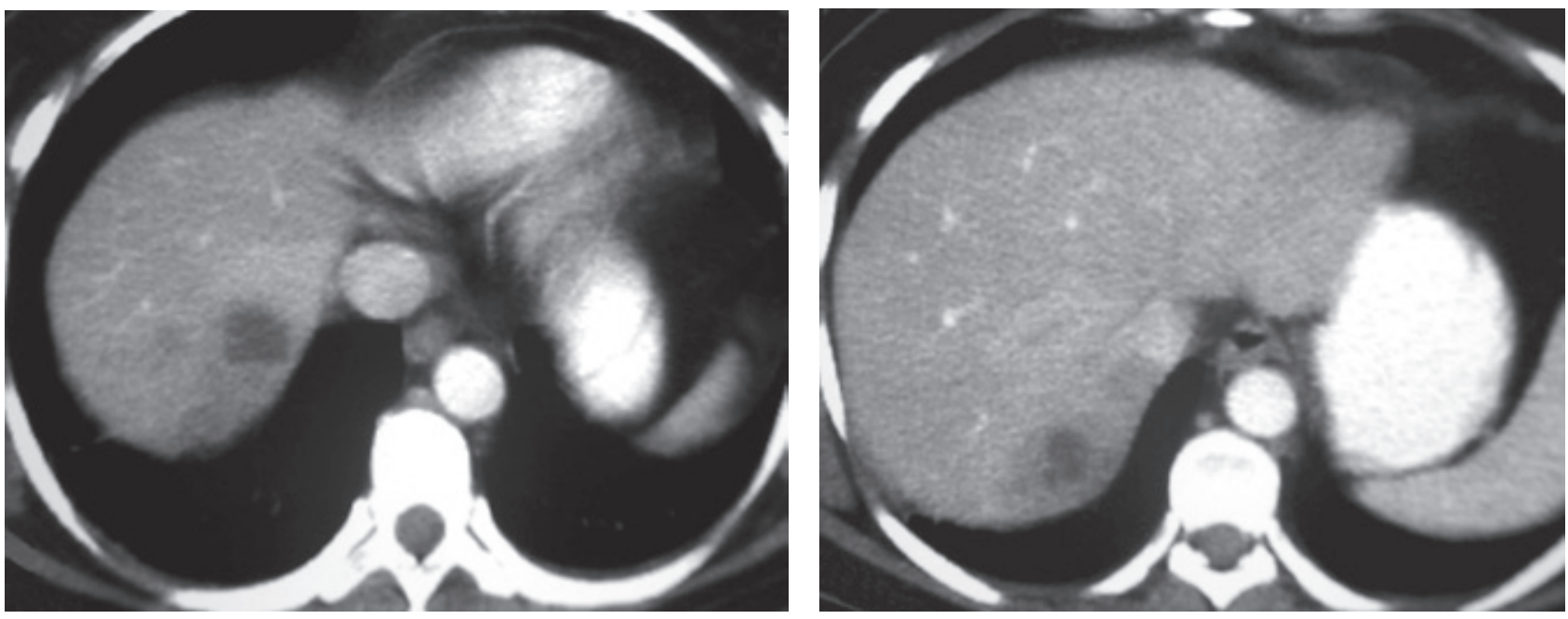

Figura 1 a,b. Tomografía computada que muestra imagen hipodensa mal definida en segmento VII, con mínimo realce periférico tras administración medio de contraste. Examen obtenido a los 10 días después de iniciado los síntomas.
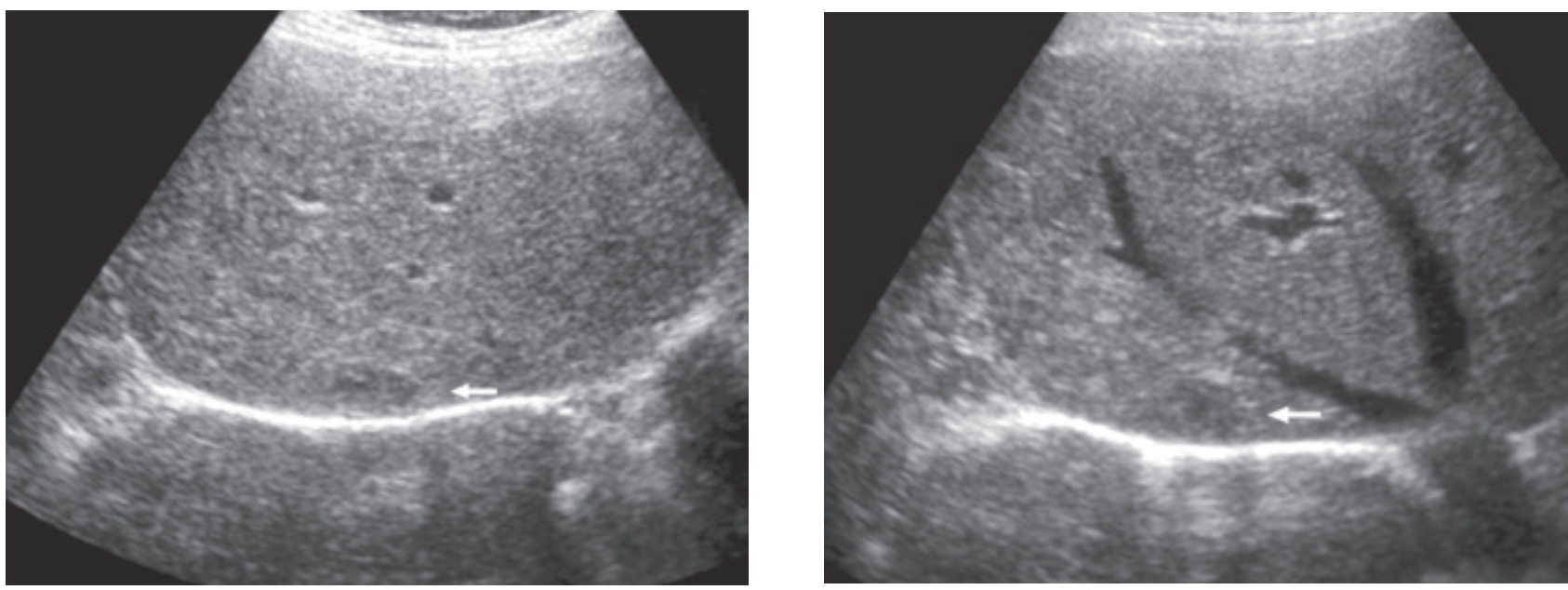

Figura 2 a,b. Ecografía que muestra tenue imagen hipoecogénica de bordes anfractuosos, periférica en segmento VII. Examen obtenido a las 3 semanas de iniciados los síntomas (Flechas).

(Figuras 3,4). Este última reveló tres lesiones hipointensas en T1, hiperintensas en T2, bien delimitadas, en relación a el segmento VII, las cuales se realzan en forma periférica con el contraste, sin llenar su espesor, incluso en cortes tardíos efectuados a los 10 minutos. Ellas varían en tamaño entre 1 y $1.5 \mathrm{~cm}$, con imágenes sugerentes de tractos. Una de ellas contacta la vía biliar.

Figura 3 a-d. Resonancia magnética secuencia T1 con imagen hipointensa en segmento VII (a).

Resonancia magnética secuencias T2 HASTE con imagenes hiperintensas en segmento VII corte axial (b) y sagital $(c, d)$.

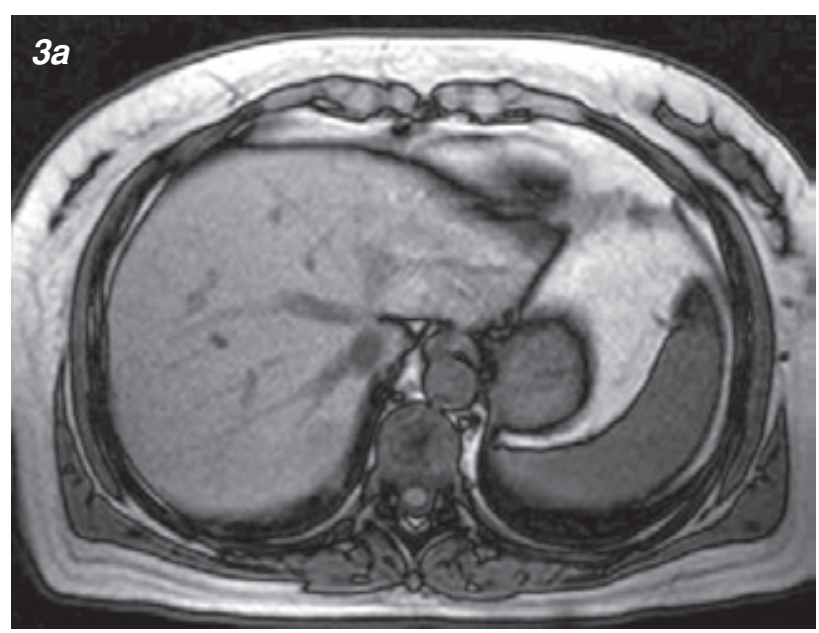



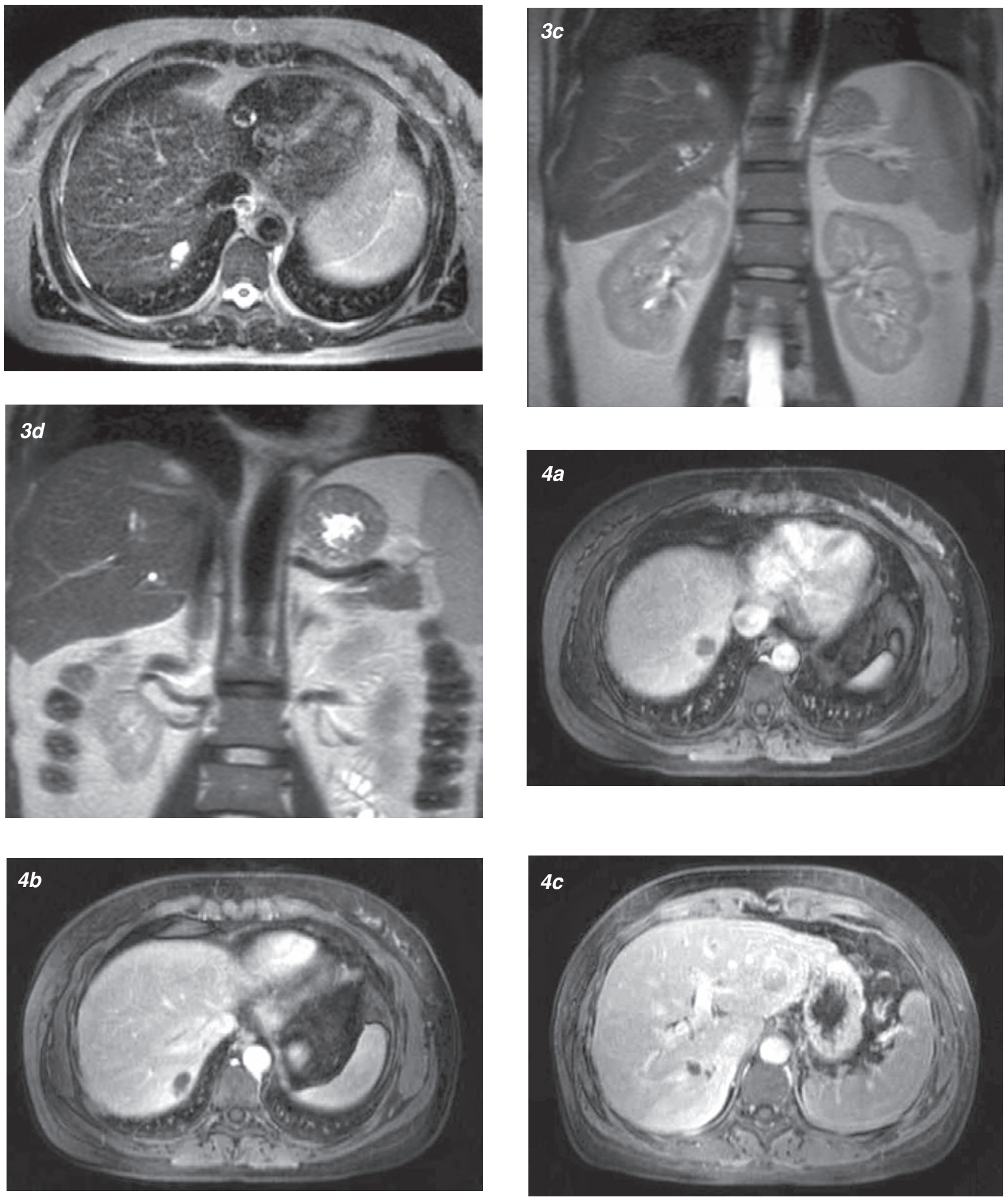

Figura 4 a-c. Resonancia magnética secuencia ponderada en T1 VIBE con administración de gadolinio en fase venosa, donde se aprecian imágenes anfractuosas, bien delimitadas, con refuerzo periférico con Gadolinio, ubicadas en segmento VII. No existe llene de ellas en cortes tardíos.

A la luz de estos hallazgos, sumado a la presencia de hipereosinofilia, se reinterroga la paciente, proporcionando el antecedente de ingesta de berros crudos aproximadamente un mes previo al inicio de los síntomas.
El diagnóstico fue confirmado con la reacción de ELISA, que fue positivo para Fasciola hepática. Se inició tratamiento con Triclabendazole, observándose en control ecográfico al mes, resolución completa de las lesiones. 


\section{Discusión}

Patogenia: (1) La Fasciola hepática, es un trematodo con aspecto de hoja alargada, que mide aproximadamente $2-3 \mathrm{~cm}$. de longitud y $1-1.5 \mathrm{~cm}$. de ancho, que en su etapa adulta se localiza en la vía biliar del ganado bovino, ovino o caprino y accidentalmente en el hombre. Su ciclo continúa al eliminarse sus huevos por las heces en agua de vertiente $o$ acequias con materia en descomposición, liberándose su primer estado larval, el miracidio. Éste debe ubicar en el plazo de 24 horas, a su huésped, un caracol de agua dulce de 5 a $10 \mathrm{~mm}$ de longitud del género Limnaea (en Chile: L. Viatrix), donde se enquista y produce su siguiente estado, la cercaria. Se calcula que por cada miracidio que llega al caracol, se producen 500 cercarias. Estas abandonan el L. Viatrix, se desplazan por el agua con su cola, hasta llegar a hierbas que crecen en ella, se enquistan nuevamente al perder su cola, y se transforman en metacercarias.

La forma de contagio en el humano se relaciona a la ingesta de verduras contaminadas, particularmente los berros (Nasturtium officinale), donde al ingresar al tubo digestivo se disuelve su cobertura y queda libre la forma juvenil.

Este distoma atraviesa la pared intestinal, cae a la cavidad peritoneal, avanza hasta llegar a la cápsula hepática, en un período aproximado de 3 a 15 días, perforándola y migrando a través del parénquima hasta llegar a los conductos biliares. En este último lugar llega a su adultez alrededor de los dos meses de la infección.

Clínica: La presentación clínica muchas veces es vaga e inespecífica, principalmente por las dos etapas de su ciclo en el humano, lo que provoca variaciones en la sintomatología. Lo inespecífico de esta es tal, que se han descrito casos de biopsias percutáneas e intervenciones quirúrgicas con otros diagnósticos, especialmente tumorales, en que solo el resultado de la biopsia ha dado el diagnóstico final(4).

Se distinguen dos fases: invasión y estado, los que se relacionan a la sintomatología.

Período de invasión: Comprende la migración al parénquima hepático y puede durar dos meses, se puede manifestar como: dolor mal definido en hemiabdomen superior, sin relación a alimentos, hepatomegalia de grado variable, fiebre intermitente de corta duración asociado a calofríos, aun cuando se han descrito casos que cursan afebril, como nuestro caso. Se ha descrito también urticaria fugaz rebelde a tratamiento.

Período de estado: Comprende la fasciola en vía biliar y puede producir: alteraciones digestivas vagas como anorexia, flatulencia, nauseas, muchas veces interpretada como "dispepsia biliar"; dolor tipo cólico biliar, ictericia transitoria, hepatomegalia, fiebre variable.

Los elementos más útiles para el diagnóstico son: la presencia de hipereosinofilia, que se observa en el $92 \%$ de los pacientes ${ }^{(5)}$, que puede ser fluctuante durante los episodios de invasión ${ }^{(1)}$, y el antecedente de ingesta de berros crudos, que esta presente en alrededor del $70 \%$ de los $\operatorname{casos}^{(3)}$.

El cuadro clínico tan inespecífico sin embargo, genera enorme dificultad en el diagnóstico clínico, siendo necesario muchas veces el apoyo imagenológico, por lo que el radiólogo debe estar preparado y conocer la epidemiología, patogenia y hallazgos en imágenes de este patógeno, especialmente en aquellas zonas endémicas.

\section{Aspectos imagenológicos}

Ellos van a depender de la fase de enfermedad en la cual se encuentre el paciente, comprendiendo desde el punto de vista imagenológico dos entidades que son la forma hepática y la biliar. En la fasciolasis hepática, su aspecto obliga a un alto grado de sospecha dado que puede ser indistinguible de otras lesiones focales hepáticas

Ultrasonido: En fasciolasis hepática, se pueden ver lesiones focales hipoecogénicas, anfractuosas, bien definidas, de tamaño variable, con tendencia a coalescer ${ }^{(6)}$ en un $96 \%$ de los $\operatorname{casos}^{(7,8)}$, aunque también se describen de ecogenicidad variable ${ }^{(9)}$. Sin embargo, no siempre es posible visualizar los trayectos que le dan el sello característico, por lo que su rol en fasciolasis aguda es menor.

Se describe también la presencia de adenopatías periportales en un $74 \%$ de $\operatorname{casos}^{(7)}$, aunque este hallazgo es inespecífico y está presente en las hepatitis aguda y crónica, neoplasias abdominales, tuberculosis abdominal y colangitis esclerosante, entre otras patologías.

En fasciolasis biliar, el rol de la US es mayor, dado que es capaz de constatar engrosamiento parietal del colédoco con dilatación moderada ${ }^{(7)}$, aun cuando este hallazgo, se ve también en patologías como colangitis esclerosante y colangitis por $\operatorname{SIDA}^{(9)}$. Más específico es la observación de estructuras móviles en vesícula biliar y/o colédoco, representando la forma adulta de fasciola ${ }^{(6-9)}$ (Figura 5). Hay autores que sugieren que el US es de menor utilidad que la TC para el diagnóstico(10), sin embargo, nosotros consideramos que su rol en fasciolasis hepática es indudable, ya que permite una evaluación más precisa de la vesícula y vía biliar extrahepática.

Tomografía computada: En fasciolasis hepática, puede presentarse como lesiones nodulares o tortuosas, con realce después de contraste variable, aunque en un $80 \%$ permite su mejor delimitación en fases tardías ${ }^{(9,11)}$. Sin embargo, lo más característico de estas lesiones es su ubicación subcapsular, con 

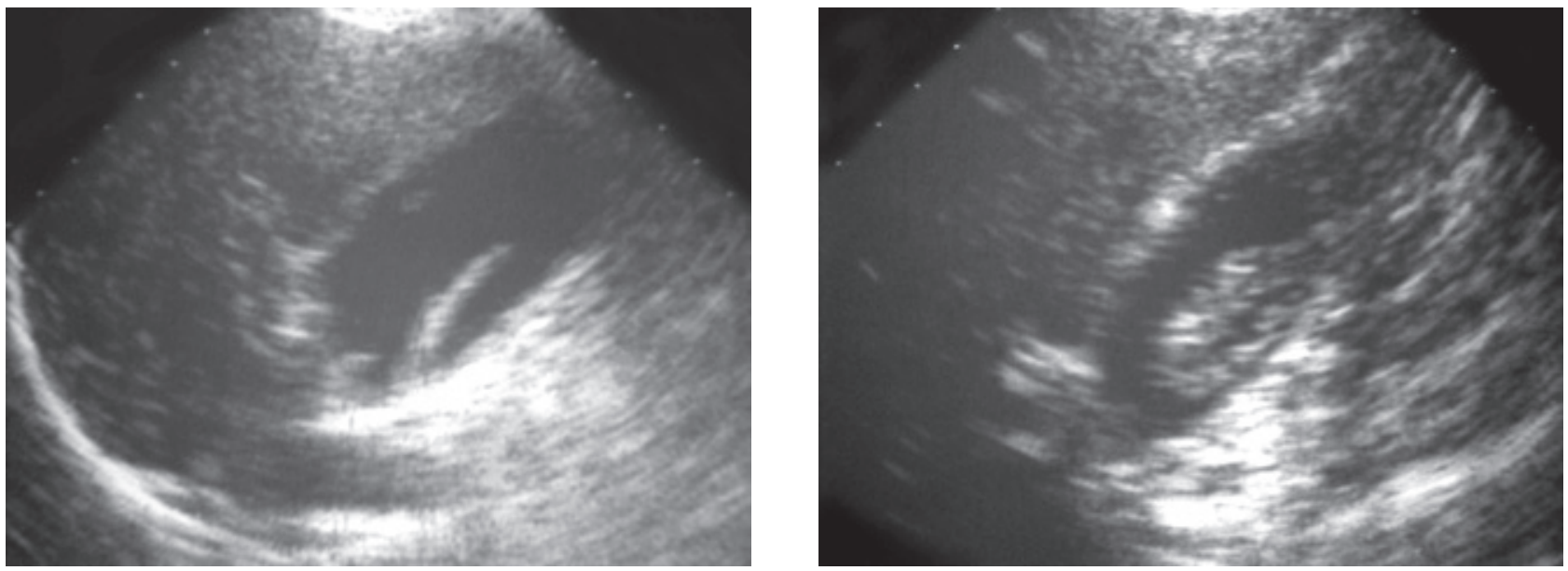

Figura 5 a,b. Vesícula biliar con Fasciola hepática en su interior. (Imágenes de archivo. Colección Dr. D. Busel)

trayectos tortuosos que adoptan una dirección centrípeta en dirección a conductos biliares intrahepáticos, cuyo aspecto hace que algunos autores lo propongan como altamente sugerente de fasciolasis ${ }^{(9)}$.

También se describe realce de la cápsula hepática con la inyección de contraste, lo que se correlaciona con engrosamiento e inflamación de ella hecho observado en laparoscopía ${ }^{(12)}$. Otro elemento a considerar es el hematoma subcapsular(13), complicación infrecuente, pero que sin duda requiere control imagenológico.

En relación a fasciolasis biliar, el rol de TC en su diagnóstico es menor, pudiendo solo mostrar mínima dilatación de vía biliar extrahepática, siendo un hallazgo no específico ${ }^{(9)}$.

Resonancia magnética: La mayor serie hasta el momento que analizó los patrones que se pueden ver en $\mathrm{RM}^{(14)}$, consistió en 29 pacientes y los clasifican de la siguiente forma.

Tipo 1: Iso o hipointensidad de la cápsula en T1, hiperintensidad de cápsula en T2, y realce de la cápsula con Gadolinio.

Tipo 2: Área iso o hipointensa en T1, áreahiperintensa mal definida en T2, con realce en forma de tractos tras administración de gadolinio.

Tipo 3: Lesión nodular hipointensa en T1, lesión nodular hiperintensa en $\mathrm{T} 2$, sin realce con gadolinio.

Tipo 4: Lesión hipointensa en T1, lesiones hipo o hiperintensas central con halo periférico hiperintenso, y realce periférico con gadolinio.

Tipo 5: Focos hipointensos o áreas mal definidas en T1 y T2, con realce de zona hipointensa con gadolinio.
También describen un $10 \%$ de los casos con RM normal y en los casos patológicos el tipo 2 $(62.1 \%)$, como el hallazgo más frecuente. Proponen que esta clasificación puede reflejar la evolución natural de esta patología, desde la invasión capsular con reacción inflamatoria (tipo 1), pasando por la fase de formación de tractos, y zonas de microabscesos inflamatorios (tipo 4 y 5). Aun más, proponen que la presencia de lesiones tipo 4 o 5 , son reflejo de enfermedad de larga data, si bien hay una importante superposición de poblaciones que impide sostener estar conclusión.

Hasta la fecha, no se han publicado estudios que utilicen medios de contraste específicos para hígado, que podrían dar más detalle del proceso de formación de abscesos y/o necrosis de coagulación asociada en esta patología, que ha sido observada en modelos animales ${ }^{(15)}$.

\section{Conclusión}

El hombre actúa como huésped intermedio y accidental de Fasciola hepática, cuando las cercarias desarrolladas en el pequeño caracol de agua Limnaea viatrix, llegan y se enquistan en verduras como los berros (Nasturtium officinale), que al ser consumidas, permite que se continúe su ciclo. En países donde la presencia del trematodo es endémico, es importante que el médico clínico, y el radiólogo en particular, lo incluyan en del diagnóstico diferencial de lesiones focales hepáticas, especialmente en el contexto clínico de eosinofilia.

\section{Bibliografía}

1. Atias A, Neghme A. "Fasciolasis" Parasitología Clínica, Tercera Edición, Capitulo 41, páginas 334-340. Editorial Mediterraneo, Santiago, Chile. 1991

2. Morales M, Luengo J, Vásquez J. Distribución y tendencia de la fasciolasis en el ganado de abasto de 
Chile. 1989-1995. Parasitol al Día 2000; 24: 115-8.

3. Apt W, Lopez X, Zulanta I, Benavente R. Fasciolasis aguda: Caso clínico. Parasitol Latinoam 2002; 57: 5558.

4. Sapunar J, Braghetto I, Diaz JC y col. Fascioliasis hepática que simularon tumores hepáticos. Bol Chil Parasitol 2001; 57 (3-4): 59-65.

5. Cosme A, Ojeda E, Cilla G Y col. Fasciolasis hepatobiliar. Estudio de una serie de 37 pacientes. Gastroenterol Hepatol 2001; 24: 375- 380.

6. Bassily S, Iskander M, Youssef FG et al. Sonography in diagnosis of fasciolasis. Lancet 1989; 1(8649): 1271 1272.

7. Kabaalioglu A, Cubuk M, Senol U et al. Fasciolasis: US, CT and MRI findings with new observations. Abdom Imaging 2000; 25: 400-404.

8. Cosme A, Ojeda E, Poch M, et al. Sonographic findings of hepatic lesions in human fascioliasis. J Clin Ultrasound 2003; 31: 358-363.

9. Van Beers B, Pringot J, Geubel A et al. Hepatobiliary fasciolasis: Noninvasive imaging findings. Radiology 1990; 174: 809-810.

10. Arjona R, Riancho JA, Aguado JM et al. Fasciolasis in developed countries: a review of classic and aberrant forms of the disease. Medicine 1995; 74: 13-23.

11. Andresen B, Blum J, von Weymarn A et al. Hepatic fasciolasis: report of two cases. Eur Radiol 2000; 10: 1713-1715.

12. Moreto M, Barron J. The laparoscopic diagnosis of the liver fascioliasis (abst). Gastrointest Endosc.
1980;26(4): 147-9.

13. Gonzalez J, Herrero A, Carrero P. Subcapsular abscess: an unusual CT finding in hepatic fasciolasis. Am J Roentgenol AJR 2002; 178: 514-515.

14. Cevikol C, Karaali K, Senol U et al. Human fasciolasis: MR imaging findings of hepatic lesions. Eur Radiol 2003; 13: 141-148.

15. Han JK, Jang HJ, Choi B et al. Experimental hepatobiliary fascioliasis in rabbits: A RadiologyPathology correlation. Investigative Radiology 1999; 34 (2): 99-108.

16. Kim K, Lim H, Kim S et al. Necrotic granuloma of the liver by human fasciolasis: imaging findings. Abdom Imaging 1999; 24: 462-464.

17. Han JK, Han D, Choi BI et al. MR findings in human fascioliasis. Trop Med Inter Health 1996; 1 (3): 367372.

18. Kamba M, Suto $Y$, Hosho K, et al. MRI appearances of fasciolasis complicating liver iron overload. $\mathrm{Br} \mathrm{J}$ Radiol 1997; 70: 1296-1298.

19. Martínez R, Ruiz J, Díaz O, y col. Diagnostico de la fasciolasis de las vías biliares por imagenología. Rev Cubana Med Trop 2000; 52 (2): 145-147.

20. Sapunar J, Latorre R, Guerra M, Defilippi C. Consideraciones clínicas a propósito de dos casos de fascioliasis hepática. Importancia de los exámenes de imágenes. Bol Chil Parasitol 1992; 47: 70-76.

21. Balci N, Sirvanci M. MR imaging of infective liver lesions. Magn Reson Imaging Clin N Am. 2002;10(1):121-135. 